\title{
High-accuracy physical layer model for wireless network simulations in NS-2
}

\author{
Jean-Michel Dricot and Philippe De Doncker
}

\begin{abstract}
While there exist many papers that compare the performances of different routing protocols for wireless ad hoc networks, these simulations are often using the same simplistic assumptions for the physical layer modeling. In this paper, we propose to combine techniques like ray tracing, Markov chains and experimental data in order to design an high-accuracy physical layer that can be implemented in the NS-2.
\end{abstract}

Index Terms-Wireless networks modeling and simulation. NS-2. Markov chains. Ray-tracing.

\section{Motivation}

W IRELESS ad-hoc network simulation is among one of the most hot topic of these ten last years. Since it is difficult to conduct experiments with real equipment and large number of nodes, computer-based simulations have always been used to investigate and to validate new routing schemes and to conduct performance analysis on future wireless networks [4] [5]. While there exist many papers that compare the performances of different routing protocols for wireless ad hoc networks, these simulations are often using the same simplistic assumptions for the physical layer modeling. In [2], the authors report that these simplistic models are more and more lowering the credibility of studies based on simulations. They also raise the difficulty to draw conclusions about the real performance of the upper layers.

Moreover, indoor wireless networks simulations using classical statistical models [7], e.g. path loss, suffer even greater lack of realism, with a direct impact on the resulting conclusions. The goal of this paper is:

1. to analyze the actual physical models implemented in the widely-used NS-2 [3] network simulator;

2. to propose a new model suitable for realistic indoor propagation modeling;

3 . to parametrize this model for simulation of $2.4 \mathrm{Ghz}$ and $5 \mathrm{Ghz}$ high-speed networks.

\section{NS-2 RADIO MODELS}

The NS-2 simulator is a discrete event simulator widely used in the networking research community [3]. It was first developped at the University of California at Berkeley and aimed at the simulation of large TCP/IP networks. It was then further extended at Carnegie Mellon University to integrate wireless extensions like IEEE 802.11, ad hoc networking and, more recently, cellular communications.

Contact: Jean-Michel Dricot, CP165/15, Université Libre de Bruxelles, 50 av. Franklin Roosevelt, 1050 Bruxelles, Belgium. E-mail:jdricot@ulb.ac.be

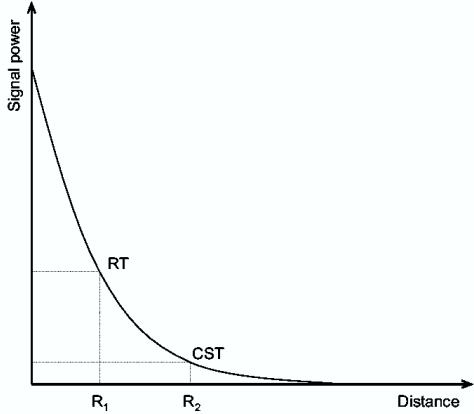

Fig. 1. Power at receiver as function of distance in NS-2 shadowing model

\section{A. Transmission area and signal strength}

The channel model in NS-2 is quite simple. The simulator computes the received power each time a packet transmission takes place by using an user-selected propagation model. Widely-used radio propagation models are the free-space model and the shadowing model.

In the first model, the world is assumed to be flat and and a direct path exists between the trasmitter and the receiver. The receiving power $P_{R x}$ depends only on the transmitted power $P_{T x}$, the gain of the transmitter and receiver $\left(G_{t}, G_{r}\right)$, the wavelength $\lambda$, the distance between both nodes $d$ and a system-wide loss coefficient $L$ :

$$
P_{R x, F S}=\frac{P_{T x} \cdot G_{t} \cdot G_{r} \cdot \lambda^{2}}{16 \cdot \pi^{2} \cdot d^{2} \cdot L}
$$

For a given simulation, only the distance may vary, the remaining of the equation being supposed to be constant. While this model is inherently limited, it is reported in [2] to be often used.

The shadowing model is an improved version of the freespace model. It considers the existence of direct and inderect rays between the two nodes and it introduces a crossover distance $d_{0}$ after which the reflecting rays may destructively interfere with the direct ray and drasticly reduce the field strength. This models also adds statistical fluctuations of the signal over time by the mean of a zero-mean Gaussian variable. The power is then computed as follows:

$$
P_{R x, S H}[d B m]=P_{R x, F S}[d B m]-n \cdot \log (d / d 0)+N(0, \sigma)
$$

where $n$ is called the path-loss exponent and $\sigma$ the shadow deviation. Both can be empirically determined and are constant for a given environment. Typical values for $n$ range from 2 (free space) to 4 (indoor) and $\sigma$ is often set to four. 


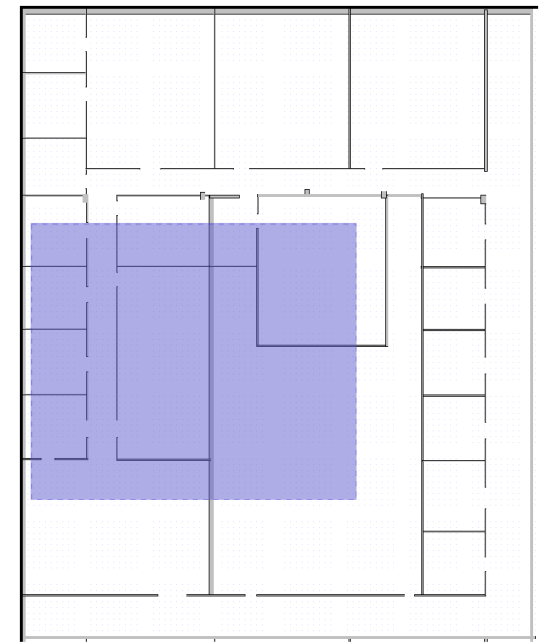

Fig. 2. Office model used for our simulations. The region of interest is inside the dashed,blue rectangle.

NS-2 makes also the assumption that the power level is also bound by two values: the receive threshold $R X_{\text {Thresh }}$ and the carrier sense threshold $C S_{T h r e s h}$ (see Figure 1). If the power falls below the $C S_{T h r e s}$, the packet is discarded as noise. If the received power level is between $C S_{\text {Thresh }}$ and $R X_{\text {Thresh }}$, the packet is marked as packet error before being captured. Otherwise, if packet is above $R X_{\text {Thresh }}$, the packet is received without errors. The consequence of this approach is the existence of circular zones for signal coverage which is incompatible with indoor deployment. Figures 2 and 3 show a typical indoor coverage. Using the shadowing model, the whole area in Figure 2 has a sufficient signal coverage, which is not verified in reality. One can easily submit to that it is impossible to parametrize the path loss model in order to fit the circular zone model with the raytracing-computed one. Moreover, the added gaussian random variable introduced to simulate the fluctuations of the communication channel has no experimental validation. Extensive measurement campaign to characterize these variations have shown that the signal deviation can be better modeled using a Rayleigh or Rice ditribution, which leads to a more severe communication channel and which has a direct influence on packet loss [1].

\section{B. Collision handling and modulation}

Once the receiver is triggered by the reception of a new packet, it checks that its present state value is idle, i.e. that it is not currently processing any incoming packet. Otherwise, the following mechanism is used:

- if the difference between the power levels of the two incoming packets is higher or equal to $10 \mathrm{~dB}$, the first packet is received and the interfering one is simply discarded,

- otherwise the medium is in collision state and both packets are lost.

Once again, this decision algorithm is too simplistic to conduct indoor wireless simulations. Indeed, when the difference between the ambiant noise and a given packet is between $0 d B$ and $10 d B$, some packets might be lost while some

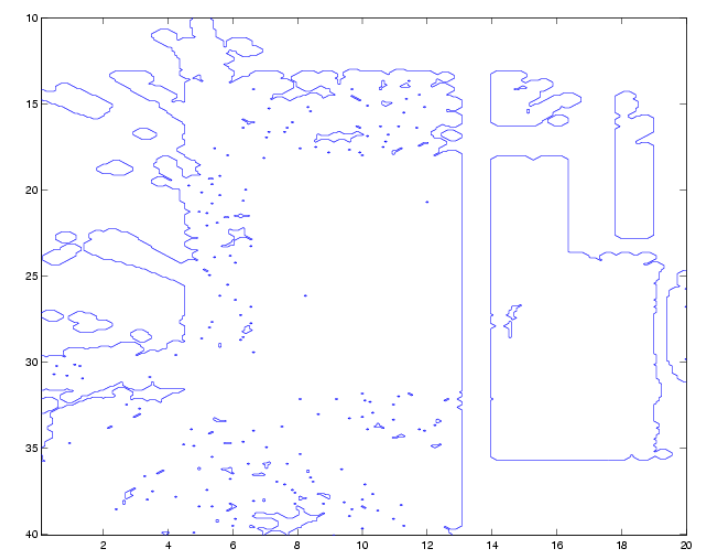

Fig. 3. Signal coverage. Emitter power is $10 \mathrm{dBm}$. The zones shown have a signal of $-70 \mathrm{dBm}$ or more, yielding a possible transmission.

others might successfully be captured. From this probabilistic behaviour a function called packet error rate is derivated. This function gives the probability of loosing a packet for a given SIR (Signal to Noise Ratio) and a given signal modulation. In NS-2 such a function is not implemented and the transmission is only two-way: totally successful $(S I R \geq 10 \mathrm{~dB})$ or totally impossible $(S I R<10 \mathrm{~dB})$.

Finally, fast variations of the signal due to people moving around are not taken into account by widely-used models. While they may have a small influence outdoor, they tend to create Doppler and large signal fluctuations with indoor operation. They will have a clear influence on the SIR especially when in presence of low-power signal. This issue will be addressed as part of the modeling of fast fading of the signal.

\section{NEW INDOOR RADIO MODEL}

In this section we propose to design a new radio model suited for indoor wireless networks simulations. Of course, not every detail needs to be introduced in the model: we have noted that furnitures and small objects have such a small influence on signal level that they can be neglected. On the other hand, walls caracteristics like permittivity and thickness should be studied in detail and the new model will be calibrated to represent a typical office situation.

The radio model we have developped is made of two parts: a ray-tracing algorithm that will be used to compute the mean signal at a given position and a Markov chain-based function aimed at reproducing the signal fluctuation that occur typically indoors. These variations are a direct consequence of people walking around and multipath fast fading.

\section{A. Average signal power computation}

Indoor wireless network efficiency is bound to the geometrical aspects of their deployment: coverage zones are never circular and rather follow the geometry of the walls and corridors of the building wherein they are deployed. 
To achieve a realistic model several techniques have been used already in deterministic or semi-deterministic models: geometrical theory of diffraction (GTD), physical optics (PO), and even some full wave methods such as integral equations (IE) or finite-difference time-domain (FDTD). Full wave methods are impossible to use here beacuse of the size of the region of interest. Such methods need mesh elements with dimensions lower than $\lambda / 8$. With the frequency of personal communications raising, these algorithm become nearly impossible to use and this is why we have choosed the GTD and PO approach.

Using GTD, the electric field at the receiver $\vec{E}_{R x}$ is computed as the sum of the fields due to each of the ray paths that connects the two nodes, i.e: direct rays, reflected rays, double reflected rays, diffracted rays, reflected-diffracted rays, etc.

$$
\vec{E}_{R x}=\vec{E}_{\text {direct }}+\sum \vec{E}_{\text {diffracted }}+\sum \vec{E}_{\text {reflected }}+\ldots
$$

The power is then inferred from the electric field at the receiver as shown in [6]:

$$
P_{R x}=\frac{1}{8 \cdot R_{a}} \cdot|-\vec{L} e \cdot \vec{E}|^{2}
$$

In this latter equation, $R_{a}$ is the antenna's resistance and $\vec{L}_{e}$ its equivalent length in the receiver's coordinates.

The computation of the received field requires then to compute every transmission, reflection or diffraction than may happen while the ray propagates from the emitter to the receiver. Prior knowledge of the environment's characteristics (permittivity $\epsilon$, conductivity $\sigma$ ) is thus required for every material (walls, doors, etc.). It can be obtained via measurement as shown below.

\section{B. Fast fading of signals}

In addition to spatial variations in signal strength, the receiver's signal level varies over time, expecially indoor when a lot of people are moving around. In most models, this variation is modeled by a Gaussian term added to the pathloss computation. This is far from adequate as it neglects the correlation of the signal over time. This correlation comes from the physical phenomenom that generates these variations is in fact a slow and permanent variation of the environment over time: people move and create hindrances, the signal paths reaching the mobile are varying with the displacement, etc.

These variations can be acquired via measurement and reproduced using Markov chains [8] [9].

A discrete time Markov chain $S_{n} \mid n=0,1, \ldots$ is a discrete time, discrete value random sequence such that given $S_{0}, \ldots, S_{n}$, the next random state $S_{n+1}$ only depends on $S_{n}$ through a transition probability

$$
\begin{aligned}
P\left[S_{n+1}\right. & \left.=j \mid S_{n}=i_{n}, S_{n-1}=i_{n-1}, \ldots, S_{0}=i_{0}\right] \\
& =P\left[S_{n+1}=j \mid S_{n}=i_{n}\right]=P_{i, j}
\end{aligned}
$$

The states we will consider here are the power level of the signal. As shown in Figure 4, a state is mapped on every signal level and a fluctuation of received power will be mapped to a transitions in the Markov chain. It is here important to note

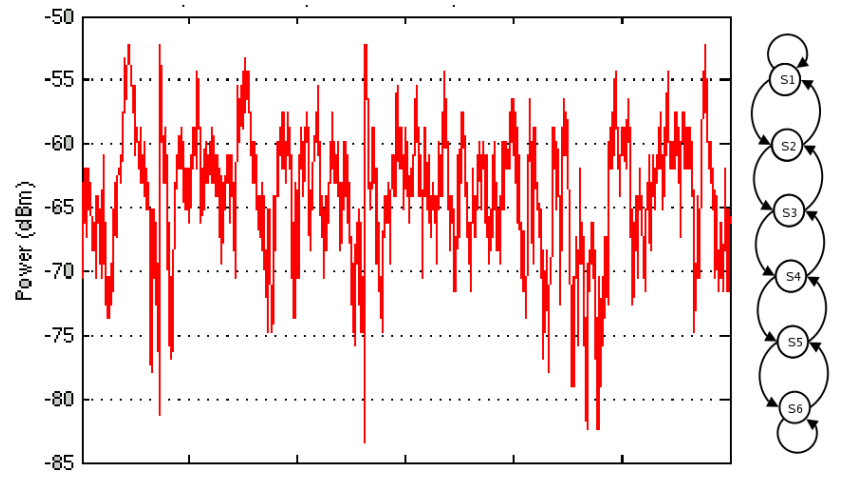

Fig. 4. Discretization of signal strength using a finite number of states.

that the Markov chain we have used here is based on the following assumptions:

1) a state of the chain represents the power of the signal in logarithmic scale $(\mathrm{dBm})$

2) during a transition, the signal strength can only move to an adjacent state

3) time interval between two transitions is constant

With these hypothesis, we can prepare a Markov chain for our simulations by choosing the number of states and a correct base time such as hypothesis 2 is respected. The transition probabilities are then found via a maximum likehood estimator: if $n_{i, j}$ denotes the number of transitions observed from state $i$ to state $j$ and $n_{i}$ denotes the total number of transitions leaving the state $i$, then the estimator is:

$$
p_{i, j}=\frac{n_{i, j}}{n_{i}} \quad i, j=1 \ldots n
$$

Finally, as the NS-2 simulator is an event-driven simulator, the question of computation of the signal level might arise at any time which seems to be contradictory with the existence of discrete time Markov chain that requires to compute a transition at regular time. This problem can be solved using the Chapman-kolmogorov theorem that gives the probability transition matrix after $n$ transitions:

$$
\text { if } \mathbf{P}=\left[P_{i, j}\right] \text { then } \mathbf{P}^{(n)}=\mathbf{P}^{n}
$$

\section{EXPERIMENTAL CALIBRATION AND VALIDATION}

The model we have implemented needs first to be calibrated in two ways:

1) the ray tracing routine computes the power for a given environment, i.e. a given permittivity and conductivity of the indoor walls,

2) the Markov chain reproduces a signal behaviour over time that first need to be captured.

The measures have been taken using an Agilent-83640B that generates a signal up to $40 \mathrm{Ghz}$. Carrier link is then acquired using a HP-8564E spectrum analyzer with three carrier frequencies: 2.4Ghz and $5 G h z$. For Markov modeling and computation of $P E R$ indoor which needs faster data aquisition, the bandwith has been set to $3.5 \mathrm{KHz}$ leading to measure rate of about 1 meas $/ 0.1 \mathrm{~ms}$. The data are then pushed into a computer using the GPIB bus and post-processed with MatLab 

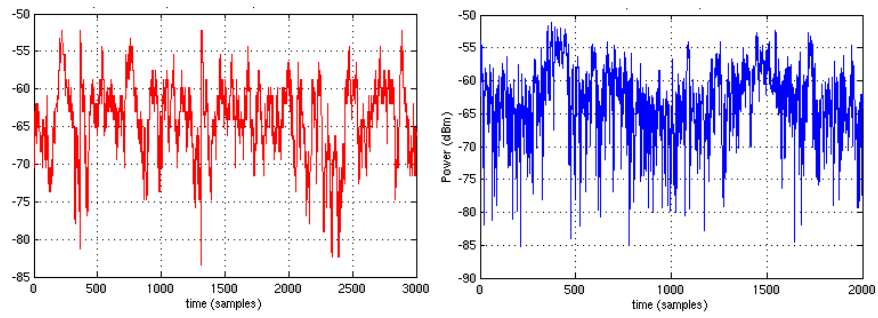

Fig. 5. Fast variations of the signal over time. The left figure shows the Markov-generated signal and the right figure shows the measured signal.

routines. The spatial variations for our model represents an office environment: people (and nodes) are moving as fast as $1 \mathrm{~m} / \mathrm{s}$. Typical decorelation distance is $\lambda / 2$, which is about $6 \mathrm{~cm}(3 \mathrm{~cm})$ at $2.4 \mathrm{Ghz}(5 \mathrm{Ghz})$. This distance cannot be travelled during a packet burst so that we can assume that each node is stationary during a whole packet transmission.

Determination of the electromagnetic parameters has been possible by measuring the decreasing signal strength along a corridor of $10 \mathrm{~m}$. The same measurement set has been simulated via the ray tracing routine using a range of values for $\epsilon: 1.0 \rightarrow 8.0$ and $\sigma: 0.1 \rightarrow 1.0$. A maximum of likehood approach has then be used to find which pair of values fits the best with the expiremental curve. For our Faculty office, we have found $(\epsilon, \sigma)=(5.2,0.8)$. It is important here to note that these two curves present the same variations but have a constant difference of level among them. This is mainly due to the desadaptation of the antennas and the loss at the entry of spectrum analyzer. This total power loss can be computed from the diference between the two curves and is about $6 \mathrm{~dB}$, which is coherent with values found in the literature.

Variation of the signal over time is two-fold: slow variations due to people moving around and fast variations due to noise and multipath. Firstly, the slowing varying enveloppe have been aquired with a sample rate of 100 samples per second. It has been found that the decorelation time for these variations is about $0.5 \mathrm{~s}$. The measured signal and the signal reproduced via the Markov chain can be found in Figures 5 and 6. The statistical parameters of the signals have been compaired to ensure that the Markov chain will give good reproduction of the reality. The mean and variance have found to be same as is the cumulative distribution function (Figure 7). Only the autocorellation functions of the two signals show a little difference but it is know in litterature [9] that Markov chains tend to have a slightly longer correlation time.

The fast variations have been measured with a sample rate sufficient to detect 10 variations of the signal's strength during a packet transmission. We have measured significative variations of signal strength and decided that they should be taken into account. PER (Packet Error Rate) is then deduced from the BER (Bit Error Rate) of the measures with the hypothesis that loosing a part of a packet leads to a whole packet failure. This means that in our simulator, packet reconstruction is not possible via some code correction technique. The failure probabilities of the parts of the packet

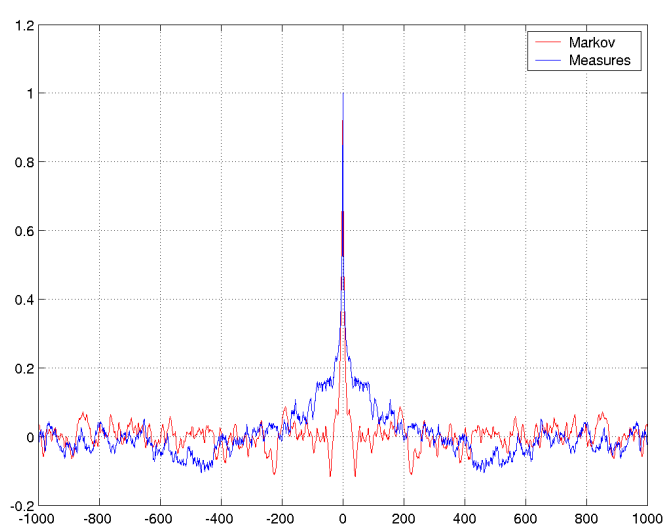

Fig. 6. Autocorrelation function of the two signals.

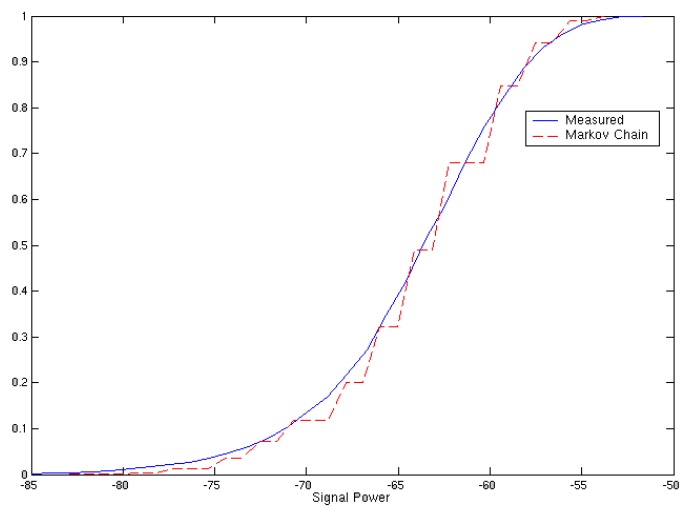

Fig. 7. DCF of the two signals.

are thus considered to be independent and we can write:

$$
1-P E R=\prod_{i=1}^{10}\left(1-B E R_{i}\right)^{L_{i}}
$$

In this equation, $B E R_{i}$ is the $B E R$ of the $i$ th sub-packet and $L_{i}$ is its length. We acquire 10 values of the signal during a packet length. The main advantage of this approach is that we do not have to consider that the noise is Gaussian, nor do we have to conduct extensive developments to compute the random variations of the signal.

\section{IMPROVEMENT IN NS-2}

The practical implementation of these two techniques (i.e. ray tracing and Markov chains) can be achived in a transparent and backward-compatible way. As the NS-2 is made of a set of $\mathrm{C}++$ or OTcl classes that enable an object-oriented approach, we have decided to implement sub-classes that will override the methods that are related to the power computation and the detection of collisions.

Firstly, the Propagation/Shadowing class has a method (namely double $\operatorname{Pr}(\ldots)$ ) which is overriden in a subclass to call the ray tracing routine in order to compute the power at receiver. When the ray tracing routine is used, a geometry file is loaded that describes the set of facets and edges present 
in building. This file can be edited via a Java editor we have designed. To avoid heavy computation, the ray tracing part of the method is re-called only after about $0.05 \mathrm{~s}$ which correspond to the decorelation time of the signal. This decorrelation time comes from that the decorelation distance of the signal is about $6 \mathrm{~cm}$ at $2.4 \mathrm{GHz}$ and that the an user travels at $1 \mathrm{~m} / \mathrm{s}$ in an indoor environment. As said before, even if the nodes ar stationary, there is a slow variation due to people moving around and a signal variation is added to the ray tracing-computed power. This signal variation is derivated from measures we have made indoor and is reproduced via a Markov chain as explained upper.

Secondly, the Modulation class has int BitError(double Power) that returns 0 or 1 depending respectively on the success or failure of the transmission due to noise or when a collision occurs. The argument of this method is the mean power during packet transmission. As $P E R$ needs knowledge of the SIR, the power of any MobileNode instance transmiting at the same time is added to the thermal noise and to the indoor noise power reproduced by our second Markov chain. As said upper, the Markov chain can reproduce the variation for a tenth of the whole packet. During the transmission, mean power (coming from ray tracing) and thermal noise can be supposed constant and are added to the value returned by the chain. For each sub-packet, we have thus a SIR and we need to find if this sub-packet is erroneous or not. The $B E R$ for each sub-packet can be obtained by using tables that give the probability of misreceiving a bit for a given $S I R$ and a given modulation. We have choosed here to use two modulations: BPSK and 16-QAM. The method then computes the probability of error of the ten sub-packets and deduces if the whole packet is successfuly received or not.

Finally, one of the most important point of the design of implementation is that these modifications must be transparent to the user, so that previous NS-2 scripts can be reused with minimal or no modifications. Thanks to OO approach of the NS-2 and to a strict inheritance/encapsulation implementation, only two lines need to be modified in the script in order to use the new propagation scheme. The former declaration

set prop [ new Propagation/Shadowing ]

has just to be replaced by

set prop [ new Propagation/Raytraced ]

sprop setFileName /foo/office.rayml

\section{CONCLUDING REMARKS}

Starting from the statement that wireless ad hoc networks simulations for indoor environment deployment needs specific tools, we have designed a new implementation that allows one to conduct fine-grained simulations with the NS-2. Wellknown techniques such as ray tracing and Markov chains are combined and the detail of their practical use is depicted. The enhanced simulator has been used to re-evaluate routing protocols like AODV,DSDV and DSR. The results of these simulations can be found in [1] and lead to results that sometimes are far from the ones obtained using the free space or shadowing models of the NS-2.
The main drawback of the new implementation is that it requires a lot of computation time. The simulations we have made required a runtime of up to 100 times longer. The drawing of the geometry files and the experimental calibration of the Markov chain for a specific indoor environment may also consume a lot of time before a set of simulations can be run. Meanwhile, these values can be reused from the ones acquired for an appreciably similar previous experience. Also, not every element of the geometries needs to be used in the simulations. As an example, we have seen that the existence of furnitures in offices does not cause a signal degradation that can modify the whole network configuration. Only walls, floors, and doors can be taken into account.

Finally, as the power of processors increase drasticly, heavy computations is less and less becoming and issue and this simulator may be used to re-run previous, well-known validations such as [10] and to raise the level of credibility of indoor wireless-related researchs and validations.

\section{ACKNOWLEDGEMENTS}

The authors would like to thank Prof. R. Meys for his help and technical support in making this work possible. The authors are also grateful to Olivier Delangre for the extensive measures he has made in order to parametrize our model.

\section{REFERENCES}

[1] J.-M. Dricot, Ph. De Doncker, E. Zimànyi, F. Grenez, Impact of the physical layer on the performance of indoor wireless networks In Proc. of the Int. Conf. on Software, Telecommunications and Computer Networks, Split, Croatia, October 2003.

[2] K. Pawlikowski, H.-D.J Jeong, and J.-S.R. Lee. On credibility of simulation studies of telecommunication networks. IEEE Communications, 40(1):132 139, January 2002.

[3] The Network Simulator. http://www.isi.edu/nsnam/ns/

[4] Per Johansson, Tony Larsson, Nicklas Hedman, Bartosz Mielczarek, and Mikael Degermark. Scenario-based performance analysis of routing protocols for mobile adhoc networks. In Proceedings of the Fifth Annual International Conference on Mobile Computing and Networking, pages 195 205. ACM Press, 1999.

[5] J. Broch, D. A. Maltz, D. B. Johnson, Y. C. Hu, and J. Jetcheva A Performance Comparison of Multi-Hop Wireless Ad Hoc Network Routing Protocols. In Proc. of the ACM/IEEE MobiCom, October 1998

[6] R.P. Meys, A summary of the transmitting and receiving properties of antennas. In IEEE Antennas and Propagation Magazine, Vol. 42 Issue 3, Jun 2000.

[7] Catedra, Manuel F., Cell Planning for Wireless Communications, Artech House, Inc, 1999

[8] Almudena Konrad, Ben Y. Zhao, Anthony D. Joseph, and Reiner Ludwig. A Markov-Based Channel Model Algorithm for Wireless Networks. In Proc. of Fourth ACM International Workshop on Modeling, Analysis and Simulation of Wireless and Mobile Systems (MSWiM 2001).

[9] Rico Schwendener. Indoor Radio Channel Model for Protocol Evaluation of Wireless Personal Area Networks. Proceedings of the 13th IEEE International Symposium on Personal, Indoor and Mobile Radio Communications, 2002 (PIMRC 2002), Sept. 2002.

[10] Josh Broch, David A. Maltz, David B. Johnson, Yih-Chun Hu, and Jorjeta Jetcheva. A Performance Comparison of Multi-Hop Wireless Ad Hoc Network Routing Protocols. In Proceedings of the Fourth Annual International Conference on Mobile Computing and Networking (MobiCom'98), ACM, Dallas, TX, October 1998. 